\title{
A RISKY ASSET MODEL WITH STRONG DEPENDENCE THROUGH FRACTAL ACTIVITY TIME
}

\author{
C. C. HEYDE, ${ }^{*}$ Australian National University and Columbia University
}

\begin{abstract}
The geometric Brownian motion (Black-Scholes) model for the price of a risky asset stipulates that the log returns are i.i.d. Gaussian. However, typical log returns data shows a leptokurtic distribution (much higher peak and heavier tails than the Gaussian) as well as evidence of strong dependence. In this paper a subordinator model based on fractal activity time is proposed which simply explains these observed features in the data, and whose scaling properties check out well on various data sets.
\end{abstract}

Keywords: Risky asset model; Black-Scholes model; heavy tails; long-range dependence; fractal activity time; self-similarity

AMS 1991 Subject Classification: Primary 90A09

Secondary $62 \mathrm{M} 10 ; 60 \mathrm{G} 18$

\section{Introduction: the basic evidence}

Over the past thirty years many risky asset models have been proposed, but these are generally deficient in not adequately explaining key features from the statistical evidence related to the issues of heavy tails and long-range dependence in financial time series. In the case of the latter, there have been problems even with understanding the nature of the characteristic, let alone its assessment.

In this note we provide a simple model which incorporates the statistical features as they are now understood. We outline the statistical evidence in Section 1. The model is given in Section 2 and its relation to the considerable previous literature is outlined in Section 3.

The paradigm model in mathematical finance is the geometric Brownian motion and this is a natural point of reference. Under this model the price $P_{t}$ at time $t$ of a risky asset is

$$
P_{t}=P_{0} \exp [\mu t+\sigma W(t)]
$$

where $\mu, \sigma^{2}>0$ are fixed constants and $W(t)$ is a standard Brownian motion. Then the corresponding log returns,

$$
X_{t}=\log P_{t}-\log P_{t-1}=\mu+\sigma(W(t)-W(t-1)),
$$

are i.i.d. Gaussian with mean $\mu$ and variance $\sigma^{2}$.

In contrast, the typical log returns data shows:

- a pronounced leptokurtic distribution (much higher peaks and heavier tails than Gaussian),

Received 12 January 1998; revision received 28 January 1998.

* Postal address: School of Mathematical Sciences, Australian National University, Canberra, ACT 0200, Australia.

Email address: chris@maths.anu.edu.au 
- a time series with high volatility and intermittency quite unlike white noise,

- strong dependence.

It is now widely appreciated that heavier tails than the normal are a necessary model feature for marginal distributions of returns. However, models with finite moments of all orders are still commonly advocated. Detailed comparisons using market index data have been given in Hurst et al. (1997) and Hurst and Platen (1997) and they favour a $t$-distribution with degrees of freedom $v$ typically in the range 3-5. This, of course, implies an infinite $k$ th moment for $k \geq v$.

Most approaches to the marginal distribution of returns have involved the use of parametric families and the choice of a best fit within a family. Few authors have investigated general questions, such as whether particular moments are finite. Part of the difficulty has been a general supposition that tail behaviour would be a power function, at least asymptotically, and that estimating the power index is problematical. Indeed, the usual method, based on the Hill estimator, is founded on order statistic theory for i.i.d. samples and it can suffer from major shortcomings outside that context. See, for example, the 'Hill Horror Plot' in Resnick (1997) and the attendant discussion.

It is, however, quite possible to test for finiteness of moments based on asymptotics obtained via the use of the ergodic theorem if the assumption of stationarity of the returns is retained. In particular, if $\sum_{i=1}^{n}\left|X_{i}\right|^{p} / n$ converges as $n \rightarrow \infty$ then $E|X|^{p}<\infty$. If, on the other hand, $\max _{1 \leq i \leq n}\left|X_{i}\right|^{p} / \sum_{i=1}^{n}\left|X_{i}\right|^{p}$ stays away from zero as $n \rightarrow \infty$ then $E|X|^{p}=\infty$. Plots as $n$ increases for large data sets should give a clear picture. For example, multiple tests on daily data from the well-known S\&P 500 index lead to the conclusion that the 4th moment is infinite (e.g. Mikosch (1997); Yang (1998)).

The next issue for modelling is that of strong dependence in the data. Log returns always display a clustering of extremes (intermittency). Sample autocorrelations of the log returns die away quickly but this is not the case for their absolute values or squares, which have non-negligible values for large lags. This is convincingly illustrated in Ding and Granger (1996) and Granger and Ding (1996) with daily returns data from the S\&P 500 index, the Nikkei index, the foreign exchange rate DM/US\$, and Chevron stock. In the case of the absolute values from the $\mathrm{S} \& \mathrm{P}$ series, for example, the lagged autocorrelations are significantly different from zero for lags up to 2700 , which is more than 10 years. The absolute values and squares of the returns exhibit long-range dependence (LRD), classically defined (for stationary finite variance processes) as holding if $\sum_{k=0}^{\infty} \gamma_{k}$ diverges, $\gamma_{k}$ being the autocorrelation at lag $k$. For a discussion of the definition and its extensions see Heyde and Yang (1997). For a comprehensive overview of the subject of LRD see Beran (1994).

Claims of long-range dependence in the log returns themselves have sometimes been made (e.g. Peters $(1991,1994)$ for the S\&P index) but this has generally been done on the basis of $\mathrm{R} / \mathrm{S}$ analysis, which is known to be highly sensitive to departures from stationarity (see for example Heyde and Dai (1996)). However, the use of other methods which are robust against departures from stationarity does not support this conclusion (Heyde and Yang (1997)). But the debate still continues. Lo (1991) developed a modified R/S statistic and on the basis of its use rejected the hypothesis of LRD in the log returns, but weaknesses in his procedure have been pointed out by Willinger et al. (1999), who dispute his assessment, albeit without compelling evidence. Nevertheless, it remains an inescapable fact that the strong dependence in the data is principally reflected in the absolute values and squares of the log returns and not in the $\log$ returns themselves.

None of the existing models capture all these features satisfactorily and we now proceed to outline how this can be done straightforwardly. 


\section{Modelling to retain the essential features of the geometric Brownian motion (GBM) model}

\subsection{Subordinator model (Stage 1)}

The $\left\{\log P_{t}\right\}$ process should have stationary differences and the absolute values and squares of the differences should exhibit LRD. We suppose that

$$
P_{t}=P_{0} \exp \left[\mu t+\sigma W\left(T_{t}\right)\right]
$$

where $\left\{T_{t}\right\}$ is a positive increasing random process with stationary differences which is independent of the Brownian motion $\{W(t)\}$ and the differences of the $\left\{T_{t}\right\}$ process are LRD and have heavy tails.

The random time changed version of GBM has all the features suggested by the data. Clock time just does not correspond to activity time (also called trading time or intrinsic market time).

Without loss of generality, choose $T_{t} \sim t$ almost surely as $t \rightarrow \infty$.

Then

$$
\begin{aligned}
X_{t} & =\log P_{t}-\log P_{t-1} \\
& =\mu+\sigma\left(W\left(T_{t}\right)-W\left(T_{t-1}\right)\right) \\
& \stackrel{\mathrm{d}}{=} \mu+\sigma\left(T_{t}-T_{t-1}\right)^{1 / 2} W(1) .
\end{aligned}
$$

The heavy tails of the $X_{t}$ come from those of $\tau_{t}=T_{t}-T_{t-1}$.

Also, for $k=1,2, \ldots$ and centred variables,

$$
\begin{aligned}
\operatorname{cov}\left(X_{t}, X_{t+k}\right) & =0 \\
\operatorname{cov}\left(\left|X_{t}\right|,\left|X_{t+k}\right|\right) & =\sigma^{2} \operatorname{cov}\left(\tau_{t}^{1 / 2}, \tau_{t+k}^{1 / 2}\right) \\
\operatorname{cov}\left(X_{t}^{2}, X_{t+k}^{2}\right) & =3 \sigma^{4} \operatorname{cov}\left(\tau_{t}, \tau_{t+k}\right)
\end{aligned}
$$

the last if $E \tau_{t}{ }^{2}<\infty$. Thus, LRD of $\left\{\left|X_{t}\right|\right\}$ and $\left\{X_{t}{ }^{2}\right\}$ follows from that of $\left\{\tau_{t}^{1 / 2}\right\}$ and $\left\{\tau_{t}\right\}$ respectively.

Other features of the model are conditional heteroscedasity,

$$
\operatorname{var}\left(X_{t} \mid \mathscr{F}_{t-1}\right)=\sigma^{2} E\left(\tau_{t} \mid \mathscr{F}_{t-1}\right)
$$

and leptokurtosis

$$
\operatorname{kurtosis}\left(X_{t}\right)=3\left(1+\operatorname{var} \tau_{t}\right)>3
$$

(if finite).

The model provides a coherent formulation for all time scales, in contrast to ARCH, GARCHtype models which focus on a single time scale.

\subsection{Subordinator model (Stage 2)}

Now we consider the activity time process in more detail, noting that it should exhibit LRD, and that LRD is often suggestive of self-similar or multifractal behaviour.

Fortunately, the activity time process $\left\{T_{t}\right\}$ can be empirically constructed. Note that the use of Itô's formula gives

$$
\mathrm{d} \log P_{t}-\frac{\mathrm{d} P_{t}}{P_{t}}=\frac{1}{2} \sigma^{2} \mathrm{~d} T_{t},
$$


from which the increments of the $\left\{T_{t}\right\}$ process may be obtained in a discretized approximation. This allows the process to be checked for self-similar or multifractal behaviour.

The kind of multifractal behaviour which might be expected is a relationship of the form

$$
T_{c t}-c t \stackrel{\mathrm{d}}{=} M(c)\left(T_{t}-t\right)
$$

for positive $c$, where $M$ and $T$ are independent random functions and $\stackrel{d}{=}$ denotes equality of finite dimensional distributions. Self-similar behaviour occurs if $M(c)$ is of the form $c^{K}$, $0<K<1$.

Approximate self-similarity can easily be checked via crude estimation of $K$. Suppose that the price $P_{t}$ is observed over a fixed time interval $[0, \mathcal{T}]$ and let

$$
S_{q}(\delta)=\sum_{i=1}^{N}\left|T_{i \delta}-T_{(i-1) \delta}-\delta\right|^{q} / N
$$

where $\mathcal{T}=N \delta$ and $0<q \leq p$ with $E T_{1}^{p}<\infty$. If $\left\{T_{t}-t\right\}$ is self-similar, then

$$
\log E S_{q}(\delta)=K q \log \delta+\log E\left|T_{1}-1\right|^{q}
$$

for $0<q \leq p$. Now the ergodic theorem ensures that $S_{q}(\delta) \stackrel{\text { a.s. }}{\rightarrow} E S_{q}(\delta)$ as $N \rightarrow \infty$, so one checks whether $\log S_{q}(\delta)$ scales linearly with both $\log \delta$ and $q$. Linearity with respect to just $\log \delta$ and not $q$ signifies multifractal behaviour.

Preliminary examination of various examples suggests that a self-similar model is usually satisfactory. For the S\&P daily returns, for example, we find $K \approx 0.8$. A comprehensive study is being undertaken.

It should be noted that the increments of the process $\left\{T_{t}-t\right\}$ can easily be shown to be LRD (SAV) for $K>1 / p$ if $E\left|T_{1}-1\right|^{p}<\infty$ for some $0<p \leq 2$ (Heyde and Yang (1997)). This nicely explains the empirically observed strong dependence. The LRD (SAV) refers to a sample Allen variance based definition of LRD which copes with processes having possible infinite variance, such as is the case here, but essentially reduces to the classical definition in the finite variance case.

For some economic discussion, based on ideas of complexity and self-organized criticality, which suggest why the activity time process (standardized to have zero mean) could have a self-similar scaling, see Arthur (1995) and Bak (1996) (particularly Chapter 11).

The principle of Occam's razor is being used here. We have sought the simplest model which reasonably represents the data, while retaining the essential features of the original GBM model.

\section{History}

A subordinated process (mixture) model formulation dates back to Mandelbrot and Taylor (1967). They used a (one-sided) stable law of index $\alpha / 2$ (where $\alpha<2$ ) for the distribution of $\tau_{t}$, which gives a symmetric stable distribution of index $\alpha$ for $W\left(T_{t}\right)$. This is theoretically attractive but does not match the data. It has infinite second moment and no LRD. Mandelbrot and Taylor conceived of the $\left\{T_{t}\right\}$ process as reflecting transaction time.

Perhaps the next was Clark (1973), who used the lognormal. This does not get the moments right and again there is no LRD. Clark related the $\left\{T_{t}\right\}$ process to trading volume. 
Many others have subsequently tried other marginal distributions. A detailed assessment of these is given in Hurst and Platen (1997). They favour the $t$-distribution with degrees of freedom in the range 3-5 on the basis of an empirical analysis of market indices for the USA, Japan, Germany, Switzerland and Australia.

Various other interpretations of the $\left\{T_{t}\right\}$ process have also been given. For example, Andersen (1996) took for his $\tau_{t}$ what he called the 'intensity of information arrivals', an unobserved process, but one which also drives the volume process.

Even though an entirely satisfactory economic model for the $\left\{T_{t}\right\}$ process may not yet be available, the characteristics of the process are fairly clear from the available statistical information.

Recent contributions containing subordinator models along similar lines to those of the present paper are Hurst et al. (1997) and Mandelbrot et al. (1997), which also contain many references to earlier literature. In the former paper the activity time process $\left\{T_{t}\right\}$ is assumed to have (stationary) independent increments. This, however, results in independent returns and does not reflect the strong dependence in observed series.

In Mandelbrot et al. (1997) it is assumed that

$$
P_{t}=P_{0} \exp \left[B_{H}(\theta(t))\right]
$$

where $B_{H}(t)$ is a fractional Brownian motion process with Hurst index $H$ and the activity time $\theta(t)$ is a multifractal process with non-decreasing paths and stationary increments. This is required to satisfy a local scaling rule of the form

$$
\theta(t+c \Delta t)-\theta(t) \stackrel{\mathrm{d}}{=} M(c)(\theta(t+\Delta t)-\theta(t))
$$

for all positive $c$, where $M(c)$ is a random variable. Now if $H=\frac{1}{2}, B_{H}(t)=W(t)$, standard Brownian motion, as used in this paper, but if $H \neq \frac{1}{2}$ the process is not a semimartingale, the standard stochastic calculus is lost, and arbitrage opportunities exist (e.g. Rogers (1997)). This makes the model unattractive for finance applications. Furthermore, if $H>\frac{1}{2}$, the returns process itself will exhibit LRD, which does not appear to be the case in practice. Also, since the increments of the positive process $\{\theta(t)\}$ are stationary, it is necessary, if the returns are to have finite first moment, that $\theta(t) \sim \alpha t$ almost surely as $t \rightarrow \infty$ for some $\alpha>0$. Hence $\theta(t)$ itself can only have a trivial scaling. Finally, in fitting the model to DM/US\$ exchange rates, Fisher et al. (1997) estimate the $H$ in their fractional Brownian motion to be 0.53 , which is close enough to 0.5 to confirm the adequacy of ordinary Brownian motion as the carrier process. They also seek multifractal behaviour for $\left\{X_{t}\right\}$, while it appears to this author that this is a property of the activity time process and not the log returns process itself.

The simple model of the present paper, which could be designated FATGBM (geometric Brownian motion with fractal activity time) captures enough of the reality of the actual processes to warrant detailed examination without obscuring the picture with additional sources of possible LRD.

\section{References}

ANDERSEN, T. (1996). Return volatility and trading volume. An information flow interpretation of stochastic volatility. J. Finance 51, 169-204.

ARThuR, W. B. (1995). Complexity in economic and financial markets. Complexity 1, 20-25.

BAK, P. (1996). How Nature Works. Copernicus. Springer, New York.

BERAN, J. (1994). Statistics for Long-Memory Processes. Chapman and Hall, New York. 
CLARK, P. K. (1973). A subordinated stochastic process model with finite variance for speculative prices. Econometrica 41, 135-155.

DING, Z. AND GRANGER, C. W. J. (1996). Modeling volatility persistence of speculative returns: a new approach. J. Econometrics 73, 185-215.

Fisher, A., Calvet, L. ANd Mandelbrot, B. (1997). Multifractality of the Deutschmark/US Dollar exchange rates. Cowles Foundation Discussion Paper No. 1165.

Granger, C. W. J. AND Ding, Z. (1996). Varieties of long memory models. J. Econometrics 73, 61-77.

HEYDE, C. C. (1997). Quasi-Likelihood and its Application. General Theory of Optimal Parameter Estimation. Springer, New York.

HEYDE, C. C. AND DAI, W. (1996). On the robustness to small trends of estimation based on the smoothed periodogram. J. Time Series Anal. 17, 141-150.

HeYde, C. C. AND YANG, Y. (1997). On defining long-range dependence. J. Appl. Prob. 34, 939-944.

Hurst, S. R. AND Platen, E. (1997). The marginal distribution of returns and volatility. In $L_{1}$-Statistical Procedures and Related Topics, ed. Y. Dodge (IMS Lecture Notes-Monograph Series 31). IMS, Hayward, CA, pp. 301-314.

Hurst, S. R., Platen, E. And Rachev, S. R. (1997). Subordinated Markov models: a comparison. Fin. Eng. Japanese Markets 4, 97-124.

Lo, A. W. (1991). Long-term memory in stock market prices. Econometrika 59, 1279-1313.

MANDElbRot, B. AND TAYlor, H. (1967). On the distribution of stock price differences. Operat. Res. 15, $1057-1062$.

Mandelbrot, B., Fisher, A. AND Calvet, L. (1997). A multifractal model of asset returns. Cowles Foundation Discussion Paper No. 1164.

MikoSCH, T. (1997). Heavy tails in finance. Bull. Int. Statist. Inst. 57, Book 2, 109-112.

Peters, E. E. (1991). Chaos and Order in the Capital Markets. John Wiley, New York.

PETERS, E. E. (1994). Fractal Market Analysis. John Wiley, New York.

RESNICK, S. (1997). Heavy tail modeling and teletraffic data. Ann. Statist. 25, 1805-1869.

Rogers, L. C. G. (1997). Arbitrage from fractional Brownian motion. Math. Finance 7, 95-105.

Willinger, W., TAqQu, M. AND TeVerovsky, V. (1999). Stock market prices and long-range dependence. Finance and Stochastics 3, 1-13.

YANG, Y. (1998). Long range dependence in the study of time series with finite or infinite variances. Ph.D. Dissertation, Columbia University, New York, NY. 